\title{
USE OF HUMAN HAZARD ANALYSIS TO ENHANCE RESILIENT PERFORMANCE OF HELICOPTER MAINTENANCE SYSTEMS
}

\author{
Simon Gill \\ The Safeguarding Community, United Kingdom
}

\begin{abstract}
When designing a new helicopter, the manufacturer must consider the maintenance tasks required to keep the aircraft airworthy with maximum efficiency. This is based on the performance of previous designs and as much as possible, the experience of real maintenance engineers bought into the team. This is rarely perfect but the system displays resilient performance, driven from the flexibility and adaptability of maintenance engineers overcoming the real-life challenges of the maintenance environment to ensure we continue to deliver safe and available aircraft.
\end{abstract}

This presentation will explore Human Hazard Analysis, a process to compare the 'Work-asImagined' in design to the 'Work-as-Done' in the real maintenance environment to reveal design and organisational limitations, but more importantly the strengths of those conducting the maintenance. The process builds upon the "Safety-I" data collected, formally and informally, on how the system has failed, but introduces a "Safety-II" focus on the workarounds developed.

We bring together groups of people from across the industry with the aim of having a different conversation. Attendees include design engineers, manufacturer technical representatives, accident investigators, experienced and novice maintenance engineers, technical directors and human factors specialists. The ground rules are simple, that openness is key. The benefit comes from honest conversations, actively avoiding the risks of defending positions, rushing to assign blame, avoiding the acceptance of blame and protecting perceived reputations.

From the insights generated we are addressing weaknesses in helicopter design and documentation, organisational norms and training, and formalising the adaptive processes employed to improve the safety and efficiency of the maintenance process.

Keywords: Human factors, Maintainability, Resilient performance, Maintenance, Human hazard analysis, Helicopter

(C) 2019 Simon Gill. This is an Open Access article distributed under the terms of the Creative Commons AttributionNonCommercial 4.0 International License (http://creativecommons.org/licenses/by-nc/4.0), permitting all non-commercial use, distribution, and reproduction in any medium, provided the original work is properly cited.

ISBN: 978-91-88898-41-8

DOI: https://doi.org/10.15626/rea8.07 


\section{INTRODUCTION}

Helicopter manufacturers design aircraft to meet specifications, aiming to find the balance between competing and often conflicting demands according to the needs of their customers. One such demand is the need to optimise the maintenance system, minimising scheduled and unscheduled maintenance and making sure that when maintenance is required that it is as efficient as possible. It has been estimated that maintenance costs account for 35-40 percent of operating costs of a commercially operated helicopter [1]. This represents a significant opportunity to save costs and as a result is a focus of effort for aircraft manufacturers. The 'maintainability' discipline integrates engineers with maintenance experience into the design process with a view to optimise maintenance tasks, including consideration of human factors principles to improve the task for the engineers [2].

Despite this effort, analysis of 'work-as-done' in the maintenance environment has consistently found that the maintenance system fails to match 'work-as-imagined' in design. There are numerous pressures faced by engineers in the course of their work which can influence their performance of the task which have been shown to contribute to this deviation. These human factors include individual (e.g. engineers physiology, or interactions with team members), environmental (e.g. lighting, noise, climate), organisational (e.g. time pressure, shift handover, safety culture), procedural (e.g. documentation) and product (e.g. required resources, accessibility of components, tooling) [3].

Maintenance engineers are routinely required to find ways of working around the discrepancies, ambiguity and dilemmas of everyday work [4]. It has been suggested that a professional subculture exists amongst aircraft maintenance technicians who hold the belief that it is their responsibility to dispatch aircraft safely and in order to achieve this, they must use their knowledge, skill and professional values [5]. Further in the same study, the authors conclude it is an accepted principle that if procedures are followed precisely they would be unable to dispatch aircraft to meet operational requirements, and that $34 \%$ of surveyed maintenance engineers had failed to perform official task procedures. Another study found $64 \%$ of maintenance engineers reported finding their own way of performing a procedure [6].

Most of the time maintenance engineers achieve the required task output. The operation continues to run, profit continues to flow, clients are satisfied, passengers get to their destination safely. Occasionally however, engineers do make mistakes but in the vast majority of cases they identify the oversight themselves. Humans have been shown to be adept at this, indeed it is a critical part of the learning process [7] [8]. If not self-identified, the maintenance system detects the mistake (through means such as duplicate or independent inspection, functional test or operational test) and it is corrected with no or little consequence. The resulting maintenance system is remarkably robust, supporting the aviation industry to achieve low accident rates and high dispatch reliability. However, to achieve this resilient performance it is the "human element that keeps [the] imperfectly designed system working effectively" [9].

In some cases this deviation does have consequences. Maintenance error has been shown to contribute to aircraft accidents and incidents, illustrated by the United Kingdom Civil Aviation Authority (UK CAA) analysis of Mandatory Occurrence Reports on large fixed wing aircraft over an extended period [10] [11] [12]. It has also been shown to result in significant cost [13] [14] and health and safety [15] consequences.

When considering the criticality of the consequences, it has been concluded in fixed wing aircraft that maintenance-related accidents are approximately 6.5 times more likely to be fatal 
than accidents in general and when fatalities do occur, maintenance accidents result in approximately 3.6 times more fatalities on average [16]. In rotary wing it was found in one study that fatal accidents accounted for $57 \%$ of all maintenance-induced failures [17]. This is explained as largely due to the low occupant survivability of rotorcraft [18].

Although most of the reported maintenance error analyses are on fixed wing civil and military aircraft, the UK CAA conducted a review of safety in offshore helicopter operations [19]. This determined that there were twenty-five UK offshore helicopter accidents between 1992 and 2013, which equates to 1.35 accidents per 100,000 flight hours or 0.66 per 100,000 flight cycle. By way of a comparison, the equivalent fixed wing accident rate was 0.4 accidents per 100,000 departures between 2000 and 2013 [20].

Helicopter accidents do have a higher criticality of accident outcomes than in fixed wing accidents. One report suggests that the number of fatalities in helicopter accidents is 10.6 times that of commercial airlines per million hours of flight [21]. This comparison does need qualification as helicopters usually operate in a more challenging environment than commercial aircraft. However, it is true to say that due to the complexity of the design of rotary wing aircraft, redundancy is more challenging (or in some cases impossible) to achieve, leading to a significantly larger number of single-point-failures than on fixed wing aircraft. In addition to this, critical parts, such as those in rotary and rotary drive systems operating under high load, represent a large proportion of the maintenance burden and are therefore more exposed to the potential for maintenance error.

These studies indicate that accidents and incidents attributed to maintenance errors continue to occur despite efforts made to address it and that these accidents tend to be more serious. Helicopter accidents occur more frequently and also tend to be more serious, and the critical parts of a helicopter are exposed to greater maintenance error potential. This represents a significant challenge, and is being robustly addressed by the offshore helicopter industry.

\section{ADDRESSING THE MAINTENANCE ERROR CHALLENGE}

The challenge illustrated here is not new, and aviation regulators have sought ways of addressing it, but their efforts have mainly focussed on the training of maintenance personnel. Changes implemented in 1999 to what is now EASA CS-66 and in 2003 to what is now EASA CS-145 required qualified engineers and all employees of organisations respectively to receive appropriate Human Factors training.

In 2006 the UK CAA proposed focusing on the design of the aircraft rather than the engineers themselves to address the challenge, suggesting regulatory change to mandate human-centred design by manufacturers of fixed wing aircraft [22]. They commissioned research to explore this approach with a number of aviation manufacturers. Airbus conducted research into a method to achieve this on fixed wing aircraft [23]. Although EASA did not pursue the regulatory change, the developed Human Hazard Analysis (HHA) process was implemented in the design process of new Airbus aircraft. Conducted by human factors specialists with designers and maintainability engineers, it aimed to identify where the maintenance system may break down to allow refinements in the design, documentation or training of engineers before the aircraft is delivered to a customer [24].

Although the approach was developed in an era of 'Safety I', where the predominant view was to manage safety by being reactive and by stopping things going wrong, this was truly proactive by addressing issues proactively in design. It also encouraged designers to consider elegant solutions to guide maintenance engineers to the correct course of action. 


\section{APPLICATION TO HELICOPTERS}

An opportunity was identified to address the helicopter maintenance error challenge outlined here building upon the HHA process developed by Airbus. This was to use HHA to analyse existing aircraft already in service (rather than new aircraft in design) to explore how successful the design had been and to identify any deviation experienced in the field between work-asdone and work-as-imagined. The source of these deviations could then be examined in detail to identify potential improvements.

A process has been developed with HeliOffshore, a global safety-focused association for the offshore helicopter industry, working with their 120 members, including operators, maintenance and design organisations for offshore helicopters, to develop a standard process for application in the industry. The focus was to adapt the academic work undertaken and the process solution implemented by Airbus fixed wing to develop a practical process for immediate use in offshore applications [25].

This Helicopter HHA process brings together a group of people who rarely have chance to work together; designers, maintainability engineers, accident investigators, technical directors, customer support engineers, human factor specialists and most importantly the engineers maintaining the aircraft on a day-to-day basis. A workshop setting is used to explore the workas-imagined by the manufacturer and work-as-done by the engineers on the front line.

\section{LINKS WITH EXISTING DESIGN ANALYSES}

\subsection{Aircraft Safety Process}

In order to assure the regulator that a new aircraft in design meets an acceptable level of safety and reliability, manufacturers in Europe utilise a safety process to comply with EASA CS25.1309 (fixed wing) [26] and EASA CS-29.1309 (rotary wing) [27]. Most adopt the framework of Aerospace Recommended Practise 4761 [28] which addresses all stages of aircraft development, from the preliminary design phase through to the in-service airworthiness monitoring after certification.

The manufacturer first defines the functions required of the aircraft and in what situations the aircraft will be unable to achieve such functions. 'Top-down' techniques such as Fault Tree Analysis determine the combinations of individual component failures which would result in those 'undesirable system states'. Coupled with component reliability targets, the manufacturer can prove that safety assurance targets have been met by the emerging design. Adherence to these reliability targets is demonstrated by component manufacturers through the use of 'bottom-up' processes such as Failure Mode and Effects Analysis. This assesses possible defects of the component being analysed, the effect of failure, how such failure would be detected and how it is being mitigated. In the design of rotary wing aircraft a process called the 'Design Assessment' is used to specify all critical components with the potential to cause catastrophic effect, especially those which originate from Single Points of Failure.

This entire process is focussed on assuring technical reliability, in order to meet certification requirements, and it does so very effectively. It also determines which components are critical to the safety of the aircraft and ensures that the level of reliability and mitigations should it fail are appropriate. What the process does not take account of however, is the variability of human interactions with the aircraft (aside from some limited common cause analyses). This is illustrated in Figure 1. 
Functional Specification of system including all functions to be achieved

\begin{tabular}{|c|c|}
\hline Process for technical functions of system & Process for human functions of system \\
\hline Basic design & Basic operating procedures \\
\hline $\begin{array}{l}\text { Specification including required degree of resilience to } \\
\text { uncontrolled factors outside system e.g. weather, } \\
\text { temperature, birds, sand }\end{array}$ & $\begin{array}{l}\text { No minimum requirements or specification for error } \\
\text { tolerance of system design during production, } \\
\text { maintenance or operations }\end{array}$ \\
\hline Technical design of system & $\begin{array}{l}\text { Recruitment \& Training specification (may be } \\
\text { incomplete e.r. engineer visual acuity standards) }\end{array}$ \\
\hline $\begin{array}{l}\text { Assess system for safety hazards from failures e.g. } \\
\text { through FHA, fault trees, FMEA and SSA }\end{array}$ & $\begin{array}{l}\text { System not assessed for safety hazards from } \\
\text { foreseeable human errors }\end{array}$ \\
\hline $\begin{array}{l}\text { Assess spotential failures modes, likely effects and } \\
\text { means of detection/recovery e.g. FMEA }\end{array}$ & $\begin{array}{l}\text { Systematic consideration of likely error types and } \\
\text { means of detection/ recovery not applied }\end{array}$ \\
\hline $\begin{array}{l}\text { Mitigate weak points that do not meet requirements } \\
\text { e.g. } 1309\end{array}$ & $\begin{array}{l}\text { Issues not identified, no standard set, therefore not } \\
\text { proactively mitigated }\end{array}$ \\
\hline Manufacture technical system & $\begin{array}{l}\text { 'Manufacture' suitable skilled humans through training } \\
\text { of pilots, engineers and production staff }\end{array}$ \\
\hline Check system work through testing/ certification & Check user competent through testing/licensing \\
\hline Monitor in service & Monitor in service \\
\hline Fix serious flaws after they occur & $\begin{array}{l}\text { Fix serious flaws after they occur (or attribute blame to } \\
\text { 'human error') }\end{array}$ \\
\hline
\end{tabular}

Figure 1 Technical vs Human Failure in Aviation Safety Processes

\subsection{Aircraft Maintenance Programme}

The Airlines for America (previously Air Transport Association) Maintenance Steering Group (MSG3) process is used to guide the development of maintenance programmes for aircraft and has been in use on fixed wing aircraft since 1968 when it was used for the new Boeing 747 aircraft [29]. A rotorcraft specific version has been developed [30] which is being used on new aircraft developed.

It uses logical decision processes to define the scheduled maintenance tasks for an aircraft and is integrated with the aircraft safety process. Common with the safety process it also fails to address the challenges of human performance, which remains a design need.

\section{HELICOPTER HUMAN HAZARD ANALYSIS PROCESS}

\subsection{Identification of Critical Maintenance Tasks}

HHA addresses the weakness in current design analyses by formalising an assessment of potential deviations from the maintenance procedures. In order to undertake such an analysis, it is important to determine the priority of where on the aircraft to focus attention. The first stage of HHA capitalises on the strength of the safety process, the identification of criticality. Using these outputs, the manufacturer identifies components which, although robustly assessed from a technical failure perspective, should be assessed from the perspective of a failure within the maintenance process. Once such components are specified, the critical maintenance tasks, 
the assumed maintenance intervals and any events that have occurred in these areas can be identified.

HHA then adopts a similar 'bottom-up' approach to the Failure Mode and Effect Analysis using a 'Human Error Mode and Effect Analysis'.

\subsection{Workshop Preparatory Work}

The makeup of the HHA team is critically important. There are core and optional team members. Core team members are a trained facilitator (ideally with human factors expertise), personnel from the manufacturer with knowledge of the design of specified components (usually designers and/or maintainability engineers) and maintenance engineers with experience of the maintenance of the specified components. Maintenance engineers of varying experience are sought, to create a combination of more junior and experienced engineers in order to bring different perspectives to the process. Other attendees who have joined the team have knowledge related to the specified components include accident investigators, customer support engineers with knowledge of in-service issues experienced by aircraft operators, and technical directors as informed observers.

All attendees are briefed in advance on the nature of the workshop and what will be asked of each of them. On the day of the workshop, setting the scene is important. When all the attendess are gathered together, the ground rules are established. There is an emphasis on the need for open, honest conversations and a need to actively change the way representatives from such organisations might historically have interacted. There must be an avoidance of defensive positions, no rush to assign blame, an acceptance of where improvements can be made and minimisation of action to protect perceived reputations.

\subsection{The Workshop Analysis}

Each component is taken in turn, with the team assessing each critical maintenance task. The team read through the steps of the task using the maintenance documentation that would be available to the engineers undertaking the task on the aircraft.

One of the first things to establish are the factors influencing the performance of the task. Airbus Helicopters undertook an analysis of maintenance errors that they had been made aware of by operators of their aircraft. This determined the following factors influencing performance of maintenance task were most significant:

- Perceived task frequency (i.e. the perception of maintenance engineers on how frequently the task must be undertaken)

- Component accessibility (i.e. visual and/or physical access to the component under investigation)

- Resource/ time needed (i.e. the number of engineers needed to perform maintenance task)

- Documentation content and clarity (i.e. the quality of the documentation provided to guide the maintenance task)

- Need for specific means (i.e. status of tooling/materials required to undertake the maintenance task).

Based on this analysis, the maintenance engineers rate the five factors from one to five, with one being low impact on performance and five being a significant impact on performance. Engineers are also encouraged to think of other influencing factors not listed. 
The team then discuss the task and any deviations from the procedure that they have experienced, observed, heard discussed in their organisations or could imagine are reasonably foreseeable. Such deviations are debated within the team to determine their reason and whether this could result in an error which risks the integrity of the design.

The team then explore if there are any control measures that exist which would address such an issue:

- Prevent Error (i.e. is there a means to prevent the maintenance error or to eliminate its consequences? e.g. the design precludes this error from occurring)

- Reduce Error Magnitude (i.e. is there a means to reduce the magnitude of the maintenance error or to reduce the magnitude of the consequences? e.g. the design is such that the consequences of this error are minor)

- Reduce Error Frequency (i.e.is there a means to reduce the frequency of the maintenance error? e.g. the conditions are favourable for the task under consideration)

- Timely Detection and Recovery within Maintenance (i.e. is there a functional, operational or other test required by the task that would detect the maintenance error and would recovery be possible? And/or is the error evident to the maintenance personnel?)

- Timely Detection and Recovery by Flight Crew (i.e. is there an indication of the maintenance error to the flight crew before flight?)

The team is then asked to rate the likelihood of control measures failing and the (immediate or subsequent) consequence should they fail on a scale from zero to five. A corresponding matrix is used determine the error sensitivity of the task. An example of such a matrix is shown in Figure 2. This is not to be used as a definitive score, but merely a means of triaging the results of the analysis, with scores to be used relative to one another.

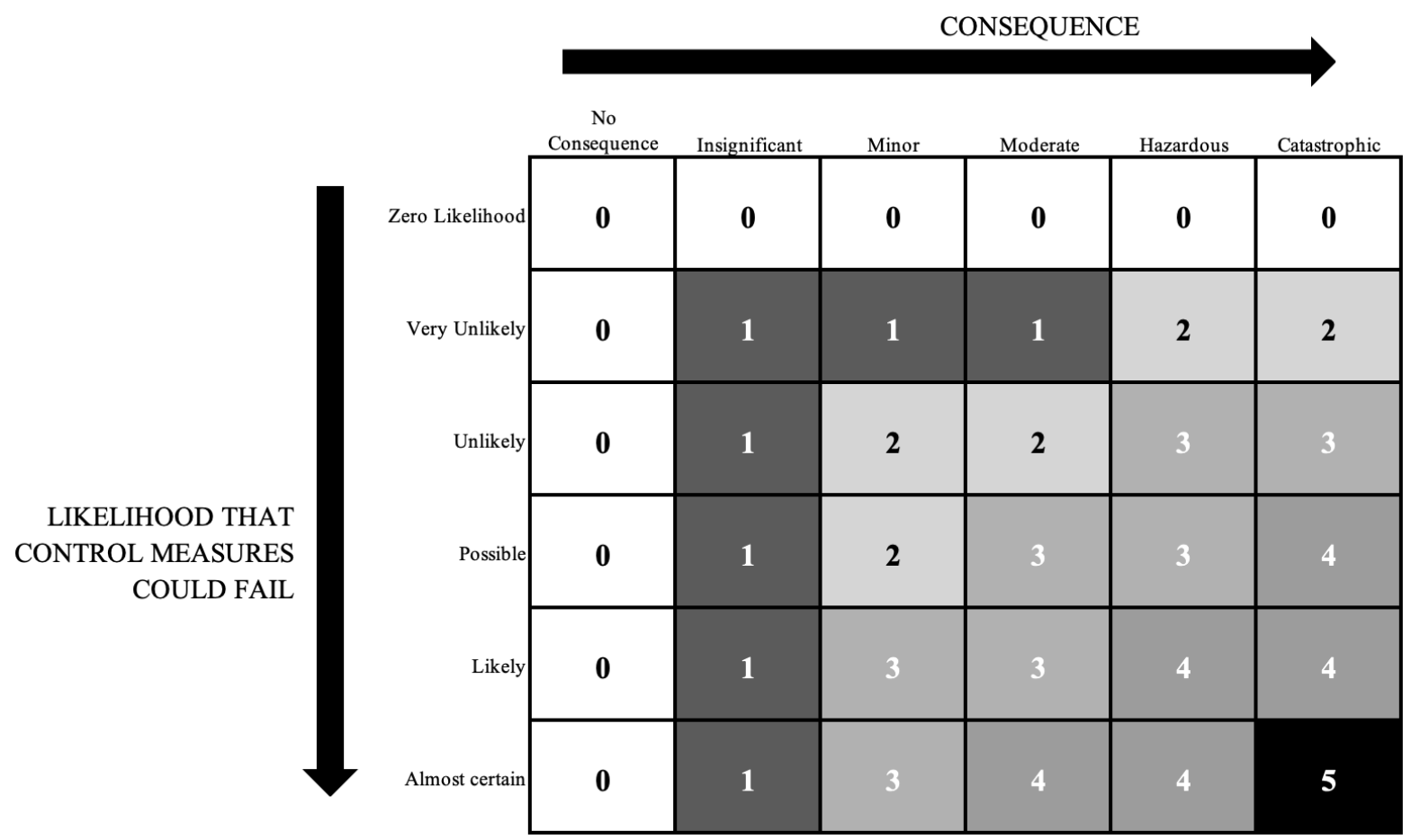

Figure 2 Example Error Sensitivity Matrix 
The team then move to identify opportunities for improvements, taking into account the previous discussions, especially what deviations from procedures are actively taking place. New control measures for errors are brainstormed, with care taken to think freely rather than limiting ideas, and to consider ideas for action by any one stakeholder in the industry - the regulator, operator, maintenance organisation or the manufacturer.

It is clear from the work conducted thus far that although the responsibility does not always lie with the manufacturer, changing the design is often the most effective means of addressing the deviation between what was originally designed and how it is used in practise. Analysis of the accident report on the loss of an AS350 near Las Vegas [31] revealed no recommendations relating to design improvements. However, as shown in Figure 3, such design changes can be simple and relatively cheap to implement [32].
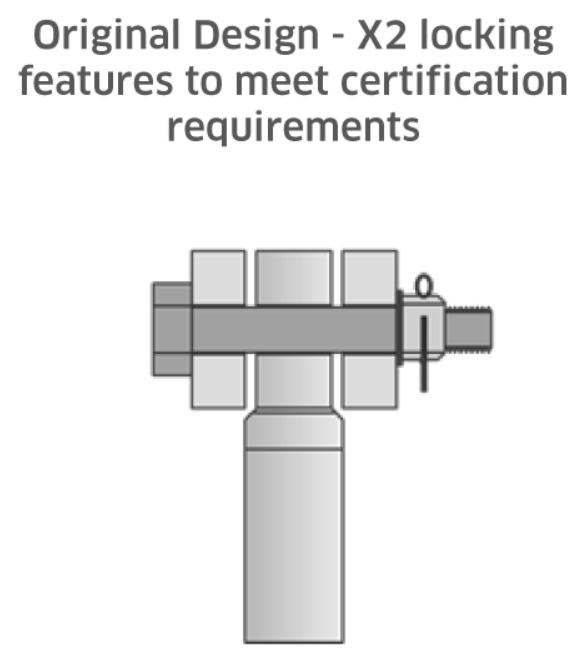

\section{Alternative Design following Human Error Modes \& Effects Analysis - X4 Locking Features}

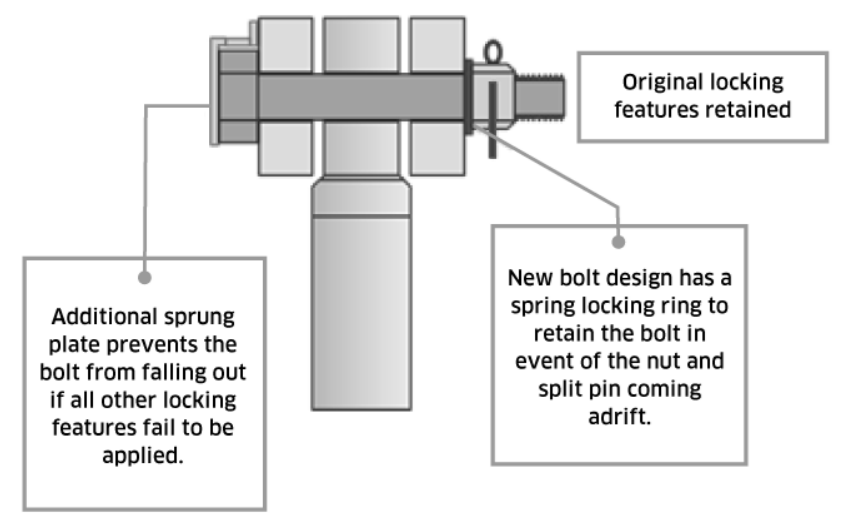

Figure 3 Additional Design Features

\subsection{Workshop Results}

Following the workshop, the manufacturer takes the specific outputs to consider short-, medium- and long-term action that they are able to take to address the identified issues. In the workshops so far, some actions have related to safety issues but there have been more which consider deviations that are not safety-specific. These have included suggestions on how documentation could be improved, and how specific processes may be changed or adapted to take advantage of the ingenuity of the maintenance engineer's approach.

Detailed here is the process designed to optimise the academic research but focussed on practicality. In its application in three locations, Norway, Italy and UK, to three different aircraft types from two aircraft manufacturers, Airbus H225, Leonardo AW139 and Airbus H175, we have seen variations in how the process has been adapted to suit design variations, organisational differences, team make-up and locality. This is to be expected and the process is designed to be flexible enough to achieve this. Equally, the use of the outputs has been different, with one manufacturer for example using it as a means of directing a detailed, on-aircraft study 
of maintenance human factors issues [33]. Another manufacturer has started using the process to assess Service Bulletins and product improvement notices before they are sent to customers.

HeliOffshore, the organiser of the workshop, takes ideas for other stakeholders to coordinate next steps and collates trends across all the workshops.

\subsection{Reported Benefits}

All participants have reported the value in the process, with the most significant outcome being the opportunity to discuss together, designer and user, the way in which the product is really used rather than as it is designed. Some specific feedback has been as follows:

- "This workshop was great to hear the opinions of the engineers. I think it is a very valuable for [the manufacturer] to have direct contact with those who maintain aircraft to test ideas, get feedback on procedures and hear about problems faced on maintaining our aircraft. It allowed us to rank maintenance tasks linked to critical parts and to give us priority for the full on-aircraft analysis to manage human factors issues." (Maintainability engineer)

- "An important part of the job of a designer is to be able to understand well how your product works, and how it will be used and perceived by end users. In this logic, operator / manufacturer transversal meetings are a super opportunity to share experience and collect precious feedback to improve ourselves and give the best aircraft for our customers." (Maintainability engineer)

- $\quad$ "It was great to be able to sit down with [the manufacturer] to allow them to hear our ideas and experience but also to allow us to hear why the aircraft was designed that way. It was a well-structured workshop to stop it being a rant from maintenance organisations, and [the manufacturer] was very open to constructive feedback in the procedures." (Maintenance engineer)

- "I was very glad to have been part of this process, allowing engineers to spend time with designers and share the problems we face. HHA is an open process to discuss problems that we would not normally be able to discuss, non-compliances and violations for example, the reality of maintenance. The HHA process broke down barriers allowing us the freedom to talk and ultimately to come up with solutions." (Maintenance engineer)

- "The open discussion was great and we moved from where we often find ourselves with the OEM in one corner and the customer is in the other - to a position where were working with one aim, to make safety improvements. We designers normally just look at theoretical steps in a maintenance task, but now I think about moving the theory to the practise, what difficulties are there that we do not normally see. It was valuable to have design, product support engineering and maintenance engineers at the same table to fix the problem quickly and we should do this more in the future." (Designer)

\section{FUTURE DIRECTION}

The offshore helicopter industry is embracing this process. Workshops are being planned on aircraft with the other aircraft manufacturers and with system manufacturers, and other design organisations. Workshops have also been requested for the aircraft which have already been subject of HHA with operators in other parts of the world. 
Taking an agile approach to process development, recommendations on enhancements to the process are implemented in subsequent workshops and feedback is constantly reviewed to ensure that the process continues to evolve positively. Software support for the developed methodology is being explored to capitalise upon the advantages of computing automation. Features such as the generation of unique reference numbers, simple copy-and-paste facilities, calculation of statistics, generation of standard reports on the progress of analysis and so on can be integrated into a software system with relative ease. Storing an archive of analyses would be useful for aircraft certification processes, for safety or quality investigations and for the review of previous analyses on similar components to guide new analyses. This would also be useful for trend analysis, for example on contributing factors and control measures. Having easy access to trends allows comparison to be made with in-service experience to assess the predictive accuracy of HHA over time, but also to justify changes in specific designs or design philosophy.

HeliOffshore is coordinating industry response on trends and lessons learnt, and communicating the results in helicopter conferences and through its membership. A Standard is being written to support a consistent approach to the process with associated training materials. Separate training is being written for design engineers to help improve their understanding of maintenance human factors and the reality of the maintenance environment, and also to integrate the findings into the mandatory training for engineers in Part 145 maintenance organisations.

\section{BIBLIOGRAPHY}

[1] Helicopter Association International cited in Ghosh, C., Maiti, J., Shafiee, M., \& Kumaraswamy, K. G. , "Reduction of life cycle costs for a contemporary helicopter through improvement of reliability and maintainability parameters," International Journal of Quality \& Reliability Management, vol. 35, no. 2, pp. 545-567. doi:10.1108/IJQRM-11-2016-0199, 2018.

[2] Bernard, F., Bazzaro, F., Sagot, J. \& Paquin, R., "Consideration of human factor in aeronautical maintainability," in 2017 Annual Reliability and Maintainability Symposium, 2017.

[3] Gill, S., "Addressing Maintenance Human Factors During Aircraft Design PhD Thesis," Nottingham University, 2009.

[4] Tsagkas, V., Nathanael, D., \& Marmaras, N., "A pragmatic mapping of factors behind deviating acts in aircraft maintenance," Reliability Engineering and System Safety, vol. 130, pp. 106-114. doi:10.1016/j.ress.2014.05.011), 2014.

[5] McDonald, N., Corrigan, S., Daly, C., \& Cromie, S., "Safety management systems and safety culture in aircraft maintenance organisations," Safety Science, vol. 34, no. 1, pp. 151-176. doi:10.1016/S0925-7535(00)00011-4, 2000.

[6] Chaparro A., Groff L. S., Chaparro B. S., Scarlett D., "Survey of aviation technical manuals. Phase 2 report : User evaluations of maintenance documentation," 2002. [Online]. Available: https://www.faa.gov/about/initiatives/maintenance_hf/library/documents/media/human_factor s_maintenance/ar02-34.pdf. [Accessed April 2019].

[7] Allwood, C., "Error Detection Processes in Statistical Problem Solving," Cognitive Science, no. 8, pp. 413-437, 1984.

[8] R., Amalberti, “The paradoxes of almost totally safe transportation systems," Safety Science, vol. 37, no. 2-3, pp. 109-126 doi:10.1016/S0925-7535(00)00045-X, 2001. 
[9] Owen, D., Gill, S., Courteney, H., McDonald, N., Liston, P., Carrera, M., "Exploring Resilience in Aviation and Maritime Transport - And How Human Factors Can Help," 2017. [Online]. Available: http://resilienceshift.org/wp-content/uploads/2017/10/026_ExploringResilience-in-Aviation-and-Maritime-Transport-And-How-Human-Factors-Can-Help.pdf. [Accessed January 2018].

[10] United Kingdom Civil Aviation Authority, "Paper 2009/05: Aircraft maintenance incident analysis," TSO, 2007.

[11] United Kingdom Civil Aviation Authority, "Paper 2007/04: Aircraft maintenance incident analysis," TSO, 2007.

[12] United Kingdom Civil Aviation Authority, “CAP1367: Aircraft maintenance incident analysis," TSO, 2016.

[13] Bourn J., "National Audit Office Report by the Controller and Auditor General- Ministry of Defence: Reliability and Maintainability of Defence Equipment," Her Majesty's Stationary Office, 1989.

[14] Hall, D., "Human Factors Training Module 1, Introduction to Human Factors in Aviation Maintenance, 15th Symposium Human Factors in Aviation Maintenance," in 15th Symposium Human Factors in Aviation Maintenance, London, 2001.

[15] DuPont, "Changing the Safety Culture of an Australian Icon - Qantas Airways Case Study," 2005.

[16] Marais, K. B., \& Robichaud, M. R., "Analysis of trends in aviation maintenance risk: An empirical approach,” Reliability Engineering and System Safety, vol. 106, pp. 104-118. doi:10.1016/j.ress.2012.06.003, 2012.

[17] Rashid, H.S.J., "Human Factors Effects in Helicopter Maintenance: Proactive Monitoring and Controlling Techniques PhD Thesis," Cranfield University, 2010.

[18] Hessmer, U., "HeliSafe ${ }^{\circledR}$ - EU research into occupant safety in helicopter crashes," Air \& Space Europe, vol. 3, no. 3, pp. 225-227. doi:10.1016/S1290-0958(01)90101-7, 2001.

[19] United Kingdom Civil Aviation Authority, "CAP1125: Civil Aviation Authority - Safety review of offshore public transport helicopter operations in support of the exploitation of oil and gas," TSO, 2014.

[20] Allianz Global Corporate \& Specialty, "Global Aviation Safety Study A review of 60 years of improvement in aviation safety," 2014. [Online]. Available: https://www.skybrary.aero/bookshelf/books/3297.pdf. [Accessed April 2019].

[21] Shell Aircraft International (2005) cited in Rashid, H., Place, S., \& Braithwaite, G., "Helicopter maintenance error analysis: Beyond the third order of the HFACS-ME," International Journal of Industrial Ergonomics, vol. 40, p. 636-647. doi:10.1016/j.ergon.2010.04.005, 2010.

[22] European Union Aviation Safety Agency, “Advance - Notice Of Proposed Amendment (NPA) No CS-25/2005," European Union Aviation Safety Agency, 2005.

[23] Lawrence, P. and Gill, S.," "Human hazard analysis: A prototype method for human hazard analysis developed for the large commercial aircraft industry," Disaster Prevention and Management: An International Journal, vol. 16, no. 5, pp. 718-739. doi:10.1108/09653560710837028, 2007.

[24] Gill, S., "Addressing Maintenance Human Factors During Aircraft Design PhD Thesis," Nottingham University, 2009.

[25] Courteney, H., Gill S., \& Carmichael S., "Designing out Maintenance Error,” March 2018. [Online]. Available: https://www.aerosociety.com/news/designing-out-human-error/. [Accessed March 2019]. 
[26] European Union Aviation Safety Agency, "Certification Specifications and Acceptable Means of Compliance for Large Aeroplanes CS-25," 2019. [Online]. Available: https://www.easa.europa.eu/regulations\#regulations-initial-airworthiness. [Accessed April 2019].

[27] European Union Aviation Safety Agency, "Certification Specifications and Acceptable Means of Compliance for Large Rotorcraft CS-29," 2019. [Online]. Available: https://www.easa.europa.eu/regulations\#regulations-initial-airworthiness. [Accessed April 2019].

[28] SAE International, “Aerospace Recommended Practise 4761,” SAE International, 1996.

[29] Airlines for America, "MSG-3: Operator/Manufacturer Scheduled Maintenance Development, Volume 1 - Fixed Wing Aircraft," Airlines for America, 2015.

[30] Airlines for America, "MSG-3: Operator/Manufacturer Scheduled Maintenance Development, Volume 2 - Rotorcraft, Rev 2015.1," Airlines for America , 2015.

[31] National Transportation Safety Board, "Loss of control Sundance Helicopters. Eurocopter AS350-B2, N37SH near Las Vegas, Nevada, December 7, 2011. Accident Report NTSB/AAR13/01 PB2013- 103890," National Transportation Safety Board, 2013.

[32] Gill, S., Carmichael S., Courteney H., "Human Hazard Analysis for Helicopters - Supporting Resilient Performance in Offshore Operations," in 12th EASA Rotorcraft Symposium, Frankfurt, 2018.

[33] Bernard F., Zare M., Sagot J-C., Paquin R., "Integration of human factors into the design process of helicopter maintainability," Hum. Factors Man. , p. 1-11.

https://doi.org/10.1002/hfm.20792, 2019. 